\title{
Study of FK Comae Berenices
}

\section{Spot evolution and detection of surface differential rotation $\star, \star \star, \star \star \star$}

\author{
H. Korhonen ${ }^{1}$, S. V. Berdyugina ${ }^{2,3}$, T. Hackman ${ }^{4}$, I. V. Ilyin ${ }^{1}$, K. G. Strassmeier ${ }^{1}$, and I. Tuominen ${ }^{4}$ \\ 1 Astrophysikalisches Institut Potsdam, An der Sternwarte 16, 14882 Potsdam, Germany \\ e-mail: hkorhonen@aip.de \\ 2 Institute of Astronomy, ETH-Zentrum, 8092 Zürich, Switzerland \\ 3 Tuorla Observatory, University of Turku, Väisäläntie 20, 21500 Piikkiö, Finland \\ ${ }^{4}$ Observatory, PO Box 14, 00014 University of Helsinki, Finland
}

Received 16 May 2006 / Accepted 18 September 2007

\section{ABSTRACT}

\begin{abstract}
Aims. We investigate the spot evolution and the surface differential rotation of the single late-type giant FK Com. Methods. A total of 18 new surface temperature maps of FK Com are calculated with the Doppler imaging technique for the years 1993-2003. Photometric observations from 2002-2004 are also given. The new and previously published spectroscopic and photometric observations are used to study the spot locations and lifetimes, and to estimate the value of the surface differential rotation. Results. The phases of the active regions determined from the Doppler images follow closely the active longitudes determined earlier from the long-term photometric observations. One active longitude can remain active for several years, but the exact spot configuration within the active longitude changes on much shorter time scales, indicating that the spot lifetime is months instead of years. There are periods during which the spot configuration changes even within days. Measurements using spot latitudes from the Doppler images and spot rotation periods from the photometric observations yield a surface differential rotation law of $\Omega=\left(151.30^{\circ} /\right.$ day $\pm 0.09^{\circ} /$ day $)-\left(1.78^{\circ} /\right.$ day $\pm 0.12^{\circ} /$ day $) \sin ^{2} \psi$ and the relative differential rotation coefficient $\alpha=0.012 \pm 0.002$ for FK Comae.
\end{abstract}

Key words. stars: activity - stars: late-type - stars: starspots - stars: individual: FK Comae Berenices

\section{Introduction}

The behaviour of the solar magnetic field can be explained by dynamo action, which is due to the interaction between magnetic fields and fluid motion. The global behaviour of the solar magnetic field can be modelled by an $\alpha \Omega$-type dynamo combined with meridional flow. In the dynamo action, the poloidal field arises from the toroidal field by helical turbulence (the $\alpha$-effect) and the toroidal field from the poloidal field by the shearing of the differential rotation (the $\Omega$-effect). Measuring the differential rotation in stars other than our Sun is very important to understand the dynamo action, and thus also the overall magnetic behaviour of stars. Theoretical calculations for G-K stars predict that the surface differential rotation becomes larger with increasing rotation period (Kitchatinov \& Rüdiger 1999), implying that for active stars, with short rotation periods, the surface differential rotation should be small.

\footnotetext{
* Based on observations obtained at the Nordic Optical Telescope, Observatorio Roque de los Muchachos, La Palma, Canary Islands, Spain; the Kitt Peak National Observatory, USA; the National Astronomical Observatory, Rozhen, Bulgaria; Automatic Photometric Telescopes Phoenix 10, Wolfgang and Amadeus, Arizona, USA.

$\star \star$ Tables $2 \mathrm{a}-\mathrm{c}$ are only available in electronic form at the CDS via anonymous ftp to cdsarc.u-strasbg.fr $(130.79 .128 .5)$ or via http://cdsweb.u-strasbg.fr/cgi-bin/qcat? J/A+A/476/881

$\star \star \star$ Figures 2 and 3 are only available in electronic form at http://www . aanda.org
}

Stellar surface differential rotation has been studied from photometric observations (e.g., Henry et al. 1995; Rodonò et al. 2000; Lanza et al. 2002), Doppler images (e.g., Donati \& Collier Cameron 1997), directly from the spectral line-profiles using the Fourier transform method (e.g., Reiners \& Schmitt 2002, 2003) and Ca II H\&K emission (Baliunas et al. 1985), of which the Doppler imaging and photometry are by far the most commonly used. By period analysis of stellar brightness variations or $\mathrm{Ca}$ II $\mathrm{H} \& \mathrm{~K}$ emission one can only determine the upper limits of the absolute value of the differential rotation coefficient. Using Doppler imaging one can, in principle, determine the whole differential rotation curve from the equator to the pole.

Doppler images obtained from separate data sets (from days to several months apart) have been cross-correlated to obtain information on spot changes in the maps. Doppler images have also been combined with time-series analysis of photometry (Hackman 2004). Using Doppler imaging several groups have reported measurements of solar-type differential rotation, i.e., that the equator rotates more rapidly than the poles (e.g., Donati \& Collier Cameron 1997; Weber \& Strassmeier 2001) and even anti-solar differential rotation (Vogt et al. 1999; Hackman et al. 2001; Hackman 2004; Weber et al. 2005). Another way of determining the surface differential rotation with Doppler imaging is to obtain the surface differential rotation pattern that minimises the information content of the reconstructed Doppler image through a simultaneous fit of all available data (Petit et al. 2002).

Cross-correlating Doppler images is a suitable method for determining surface differential rotation if the observations are 
of good quality and the maps are reliable, i.e., when the data has good signal-to-noise ratio $(S / N)$, high spectral resolution, there are no large gaps in the phase coverage and the exposure time is short enough so that no significant phase smearing happens during it. Unfortunately, often the resolution of the maps does not allow for an accurate measurement of the differential rotation. A more serious problem is the artifacts caused by systematic errors in the line modelling, phase gaps and low $S / N$ observations. The artifacts tend to appear as vertical stripes or arches in the images. If these are included in the cross-correlation a real differential rotation may be undetected. Furthermore, the spots are usually concentrated in a small latitude range, which makes an accurate determination difficult. An additional problem is that it is difficult to obtain observing runs close in time. Often the maps used in the surface differential rotation studies have a long separation in time, sometimes even a year, which affects the accuracy of the result and only gives reliable results if the active regions seen in the two maps are really the same.

Here we analyse all available spectroscopic and photometric observations of the prototype of the small group of FK Comae type stars (Bopp \& Rucinski 1981). FK Com is a single, extremely rapidly rotating, late type giant with $v \sin i \approx$ $160 \mathrm{~km} \mathrm{~s}^{-1}$ (see e.g., Huenemoerder et al. 1993). FK Com exhibits very strong photospheric and chromospheric activity (e.g., Merrill 1948; Chugainov 1966; Ramsey et al. 1981; Dorren et al. 1984; Oliveira \& Foing 1999). FK Com is also the first star where the so-called flip-flop phenomenon was found (Jetsu et al. 1991, 1993, 1994b): the spots concentrate on, and switch between, two active longitudes that are $180^{\circ}$ apart. The main activity switches longitude every 2-3 years, which is similar to the flip-flop cycles discovered in RS CVn-type stars (Berdyugina \& Tuominen 1998). The surface structures of FK Com have been extensively studied by Doppler imaging (Piskunov et al. 1994; Korhonen et al. 1999, 2000, Papers I and II; Korhonen et al. 2001b) revealing mainly high latitude spots. In this study the spot evolution and the surface differential rotation on FK Com are investigated.

\section{Observations}

In this paper new photometric and spectroscopic observations of FK Com are published and analysed together with previously published observations. The phases for all the observations were calculated using the ephemeris obtained from 25 years of photometric observations, HJD $=2439252.895+(2.4002466 \pm$ $0 \mathrm{~d} 0000056) \mathrm{E}$, referring to a photometric minimum and period calculated by Jetsu et al. (1993, 1994a).

\subsection{Spectroscopy}

In total we present 25 Doppler images of FK Com, of which 18 are previously unpublished. The spectroscopic data and Doppler images from August 1994-January 1998 were published in Papers I, II \& Korhonen et al. (2001b). Some of the 1998-2003 maps were presented in Korhonen et al. (2004) without giving observational details, so in this paper they are considered as new observations. The new data were obtained at: the Nordic Optical Telescope (NOT) in La Palma, Spain; the Kitt Peak National Observatory (KPNO) in Arizona, USA and the National Astronomical Observatory (NAO) in Rozhen, Bulgaria.

The NOT observations were obtained using the SOFIN highresolution échelle spectrograph with the low and medium resolution cameras. For the medium resolution camera the échelle spectra typically consisted of 14 orders and were centred at
$6427 \AA$, and in the case of the low resolution camera of 45 orders and which were centred at $5200 \AA$.

The KPNO observations were obtained at the 0.9-m Coudé feed telescope. The F3KB CCD detector was employed together with grating $\mathrm{A}$, camera 5 and the long collimator. The useful wavelength range was $300 \AA$ centred at $6500 \AA$.

The NAO observations were obtained with the Coudé spectrograph at the $2 \mathrm{~m} \mathrm{RCC}$-telescope. The observations were centred at $6420 \AA$ and the useful wavelength range was $100 \AA$.

The reductions of the NOT and NAO data were done using the 4A reduction package (Ilyin 2000). The KPNO data was reduced with the Image Reduction and Analysis Facility (IRAF) distributed by KPNO/NOAO.

The spectroscopic observations are summarised in Table 1. In this table the time of the observations is given together with other details. In most cases, the observations taken during the same night immediately after each other were averaged to increase the $S / N$ of the individual phases. In Table 1 the exposure time, given in minutes, is the the total time range used for one individual phase, i.e., after some spectra have been combined. However, no spectra were combined so that the total exposure time exceeded $75 \mathrm{~min}$, which corresponds to a rotation of 0.02 in phase. In the case of some old observations the exposure time of the individual spectra exceeded the limit of $75 \mathrm{~min}$. The exposure times for the June 1993 observations were between 60 and 120 min, meaning that the corresponding Doppler image was not considered reliable.

\subsection{Photometry}

For the years 2002-2004 new automatic photometric telescope (APT) observations were obtained between January 1, 2002 and July 8, 2004 using the Phoenix 10, Wolfgang and Amadeus APTs in Arizona, USA. The observations are published in Tables 2a-c. These tables are available at the CDS, and they contain the Julian Date of the observations and the observed magnitudes: $U, B$ and $V$ for Table 2 a (Phoenix 10), $V$ and $I_{\mathrm{c}}$ for Table $2 \mathrm{~b}$ (Amadeus), and $b$ and $y$ for Table 2c (Wolfgang).

These photometric observations were used in the analysis together with the previously published data by Jetsu et al. (1993), Strassmeier et al. (1997) and Korhonen et al. (2001a, 2002). The data were obtained and reduced in the same way as described in Strassmeier et al. (1997) and Korhonen et al. (2001a) and are summarised in Table 3.

\section{Temperature maps}

The surface temperature maps for this paper were calculated using the Tikhonov Regularization inversion code INVERS7PD, written by Piskunov and modified by Hackman (Hackman et al. 2001). The stellar parameters adopted for Doppler imaging can be found in Table 4. More details on the selection of the parameters, inversion technique and line-profile calculation were given in Papers I and II. In the inversions the whole spectral region from $6416 \AA$ to $6444 \AA$ was used simultaneously, as this wavelength range was obtained in a single order in the SOFIN medium resolution camera image. The atmospheric line at $6433 \AA$ was masked out from the inversions.

\subsection{Results}

All the temperature maps for 1993-2003 are plotted in Fig. 1, and the fits to the spectra and $V$ band photometry are published 
Table 1. Summary of the previously unpublished spectroscopic observations of FK Com. The table gives the name of the data set, the Heliocentric Julian Dates and the dates of the observations, the number of nights with observations, number of individual spectra, the total exposure time of the individual phases, resolving power of the instrument and the observatory at which the observations were obtained.

\begin{tabular}{lccccccc}
\hline \hline Data set & $\begin{array}{c}\text { HJD } \\
2400 \text { 000+ }\end{array}$ & Date & Nights & Spectra & $\begin{array}{c}\text { Exp } \\
{[\mathrm{min}]}\end{array}$ & $\begin{array}{c}\text { Resolution } \\
(\lambda / \Delta \lambda)\end{array}$ & Telescope \\
\hline June 1993 & $49140.4-49168.4$ & $01.06-07.07 .93$ & 7 & 7 & $60-120$ & $75000 / 30000$ & NOT/NAO \\
March 1994 & $49433.4-49446.4$ & $21.03-03.04 .94$ & 12 & $94^{*}$ & $10-60$ & 30000 & NAO \\
March 1998 & $50882.6-50892.8$ & $10.03-20.03 .98$ & 10 & 28 & $30-60$ & 51000 & NOT \\
April 1998_1 & $50904.9-50913.8$ & $01.04-10.04 .98$ & 9 & 9 & $45-75$ & 27000 & KPNO \\
April 1998_2 & $50914.8-50925.9$ & $11.04-22.04 .98$ & 10 & 10 & 75 & 27000 & KPNO \\
July 1998 & $50999.4-51010.4$ & $02.07-15.07 .98$ & 13 & 38 & $20-60$ & 27000 & NOT \\
April 1999 & $51295.4-51307.4$ & $26.04-08.05 .99$ & 7 & 18 & $20-40$ & 30000 & NAO \\
May 1999 & $51323.4-51333.6$ & $24.05-04.06 .99$ & 12 & 37 & $15-45$ & 27000 & NOT \\
July 1999 & $51383.4-51394.4$ & $23.07-03.08 .99$ & 12 & 26 & $30-50$ & 27000 & NOT \\
March 2000 & $51632.8-51669.8$ & $29.03-05.05 .00$ & 20 & 20 & $65-75$ & 27000 & KPNO \\
April 2000 & $51648.3-51652.5$ & $13.04-17.04 .00$ & 4 & 14 & $25-65$ & 30000 & NAO \\
August 2000 & $51764.4-51775.4$ & $07.08-18.08 .00$ & 12 & 30 & $30-40$ & 27000 & NOT \\
May 2001 & $52036.5-52038.7$ & $06.05-09.05 .01$ & 3 & 14 & $30-60$ & 27000 & NOT \\
June 2001 & $52064.4-52072.5$ & $03.06-12.06 .01$ & 9 & 34 & $30-40$ & 27000 & NOT \\
August 2002 & $52507.4-52516.4$ & $20.08-29.08 .02$ & 8 & 13 & $20-30$ & 27000 & NOT \\
November 2002 & $52591.8-52601.8$ & $13.11-23.11 .02$ & 5 & 6 & $10-30$ & 27000 & NOT \\
June 2003_1 & $52794.4-52801.4$ & $04.06-10.06 .98$ & 7 & 8 & $10-15$ & 27000 & NOT \\
June 2003_2 & $52802.4-52813.4$ & $11.06-23.06 .98$ & 6 & 7 & $5-11$ & 27000 & NOT \\
\hline Of 2500
\end{tabular}

* Of the 94 spectra only 75 are usable. The spectra from March 21-24, 1994 are affected by a very strong interference pattern.

Table 3. Summary of the photometric observations of FK Com for the years 2002-2004. In the table the Heliocentric Julian Dates and the dates of the observations are given, together with the number of observations with each filter and the name of the telescope where the observations were obtained.

\begin{tabular}{llccccccl}
\hline \hline HJD 2 400 000+ & Date & $U$ & $B$ & $V$ & $I_{\mathrm{c}}$ & $b$ & $y$ & Telescope \\
\hline 52 275.9-53 194.7 & $01.01 .02-08.07 .04$ & 451 & 513 & 493 & - & - & - & Phoenix 10, APT \\
52 278.0-53 185.7 & $03.01 .02-29.06 .04$ & - & - & 498 & 433 & - & - & Amadeus, APT \\
$52278.0-53172.8$ & $03.01 .02-16.06 .04$ & - & - & - & - & 115 & 107 & Wolfgang, APT \\
\hline
\end{tabular}

Table 4. Adopted stellar parameters for Doppler imaging.

\begin{tabular}{ll}
\hline \hline Parameter & Adopted value \\
\hline$T_{\text {eff }}$ (unspotted) & $5000 \mathrm{~K}$ \\
$\log g$ & 3.5 \\
Period & 2.4002466 \\
$v \sin i$ & $159 \mathrm{~km} \mathrm{~s}^{-1}$ \\
Inclination & $60^{\circ}$ \\
Microturbulence & $1.0 \mathrm{~km} \mathrm{~s}^{-1}$ \\
Macroturbulence & $2.0 \mathrm{~km} \mathrm{~s}^{-1}$ \\
\hline
\end{tabular}

electronically in Figs. 2 and 3, respectively. Some of the new maps, but without the fits to the spectra, were already presented in Korhonen et al. (2004). In the case of the April 1998 KPNO observations, two separate maps were obtained from the observations spanning 20 nights. This was done due to the rapidly changing light-curve of FK Com during 1998. A summary of the properties of the new Doppler images is given in Table 5. In this table the name of the data set is given together with the information on the phase coverage, inversions, temperature range and spot locations.

The spot locations were determined from the Doppler images as areas where the temperature is cooler than a limiting temperature $T_{\text {lim }}$. To allow also penumbra-like structures and more loosely bound spot groups that might be seen as less contrast active regions to be included in, the limiting spot temperature was set to $T_{\text {lim }}=4500 \mathrm{~K}$. This limiting temperature is based on the observations of the temperature difference between sunspot penumbra and the solar photosphere. The temperature structure in the penumbra is highly varying, depending on the exact feature and height, but the coolest temperatures found in the dark filaments are approximately $750 \mathrm{~K}$ less than that of the unspotted surface and the warmest temperatures in the bright filaments are approximately $250 \mathrm{~K}$ less than the unspotted surface (see e.g., Bellot Rubio et al. 2006). Here, a $500 \mathrm{~K}$ difference is chosen as the exact contribution of the bright and dark filaments in the stellar penumbra is not known. In Doppler imaging the changing phase coverage can affect the contrast between the spots and the unspotted surface. The data sets used here have different spacing for the phases, and this can cause a small effect on the relative contrast between the spots in different images.

In most cases the inversions were done using simultaneous $V$ band photometry as a constraint. A very small weight is given to the photometry during the inversions: $0.01-0.05$, and in the majority of the cases $0.01-0.02$. The exact value for each map is calculated by $\mathrm{No}_{\mathrm{ph}} /\left(\mathrm{No}_{\mathrm{sp}} * \mathrm{No}_{\mathrm{wl}}\right)$, where $\mathrm{No}_{\mathrm{ph}}$ is the number of photometric phases, $\mathrm{No}_{\text {sp }}$ the number of spectroscopic phases, and $\mathrm{No}_{\mathrm{wl}}$ the number of wavelength points $(\sim 100)$ contributing to each Doppler image. The weight for the spectroscopy is 1. For all the good quality spectra, maps without photometry have also been calculated. The results are close to the results obtained when using photometry as a constraint. For consistency, in this paper only the maps where the photometry has been used as a constraint are shown, if simultaneous or near simultaneous photometry is available. For two data sets (August 2000 and August 2002) there were no simultaneous photometric observations available, and for these maps the photometry was not used as a constraint; also for the maps published in Papers I and II 

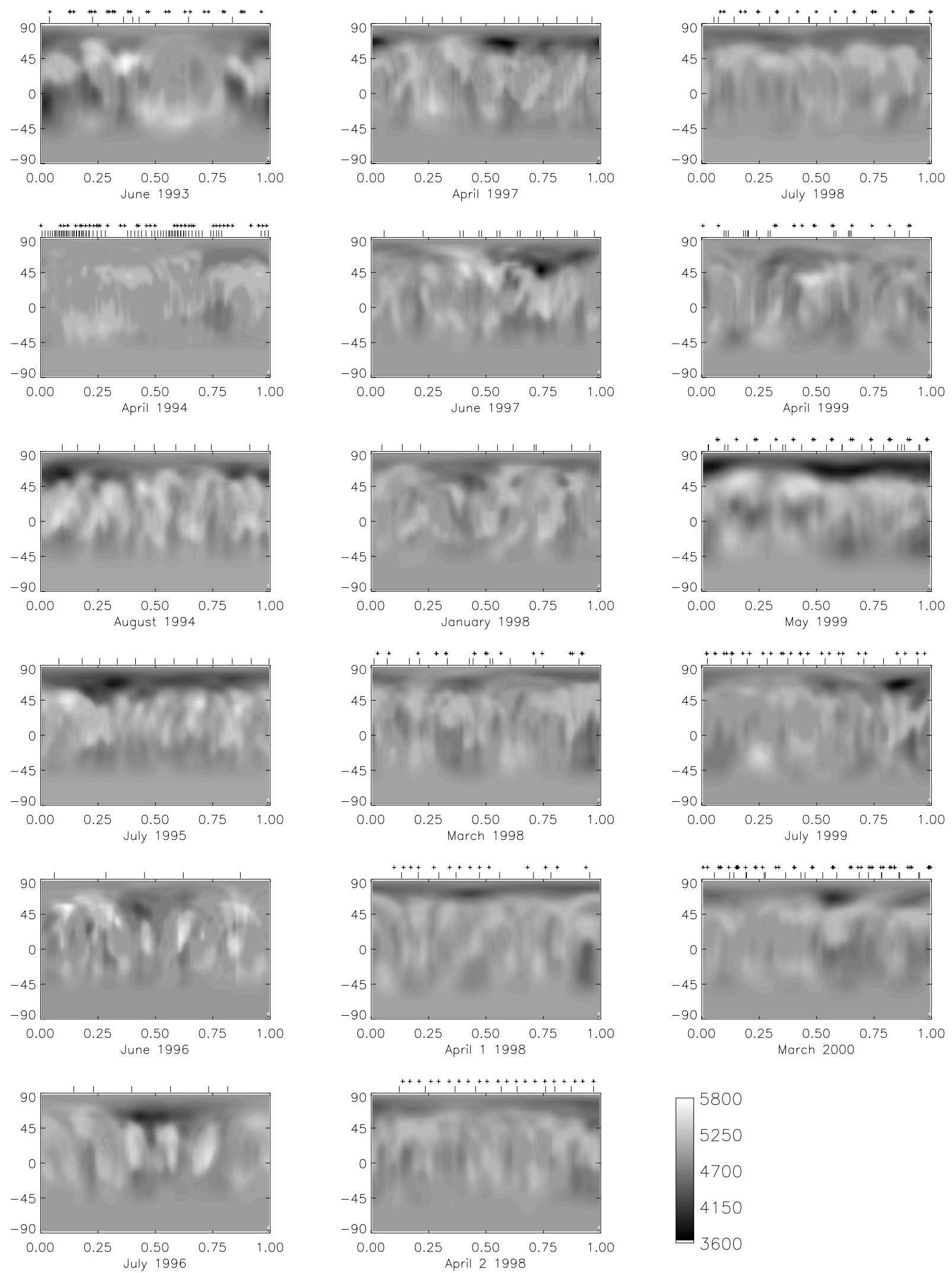

Fig. 1. Doppler images from the years 1993-2003. The abscissa is the longitude in phases and the ordinate is the latitude in degrees. The crosses above the plots denote the phases of the photometric observations and the dashes the phases of the spectroscopic observations used in obtaining the temperature map. For the August 2000 and August 2002 data sets there were no simultaneous photometry available. The August 1994 and July 1995 maps are taken from Paper I, the maps for 1996 and 1997 from Paper II and the January 1998 map from Korhonen et al. (2001b). Photometry was not used as a constraint in these previously published temperature maps. 

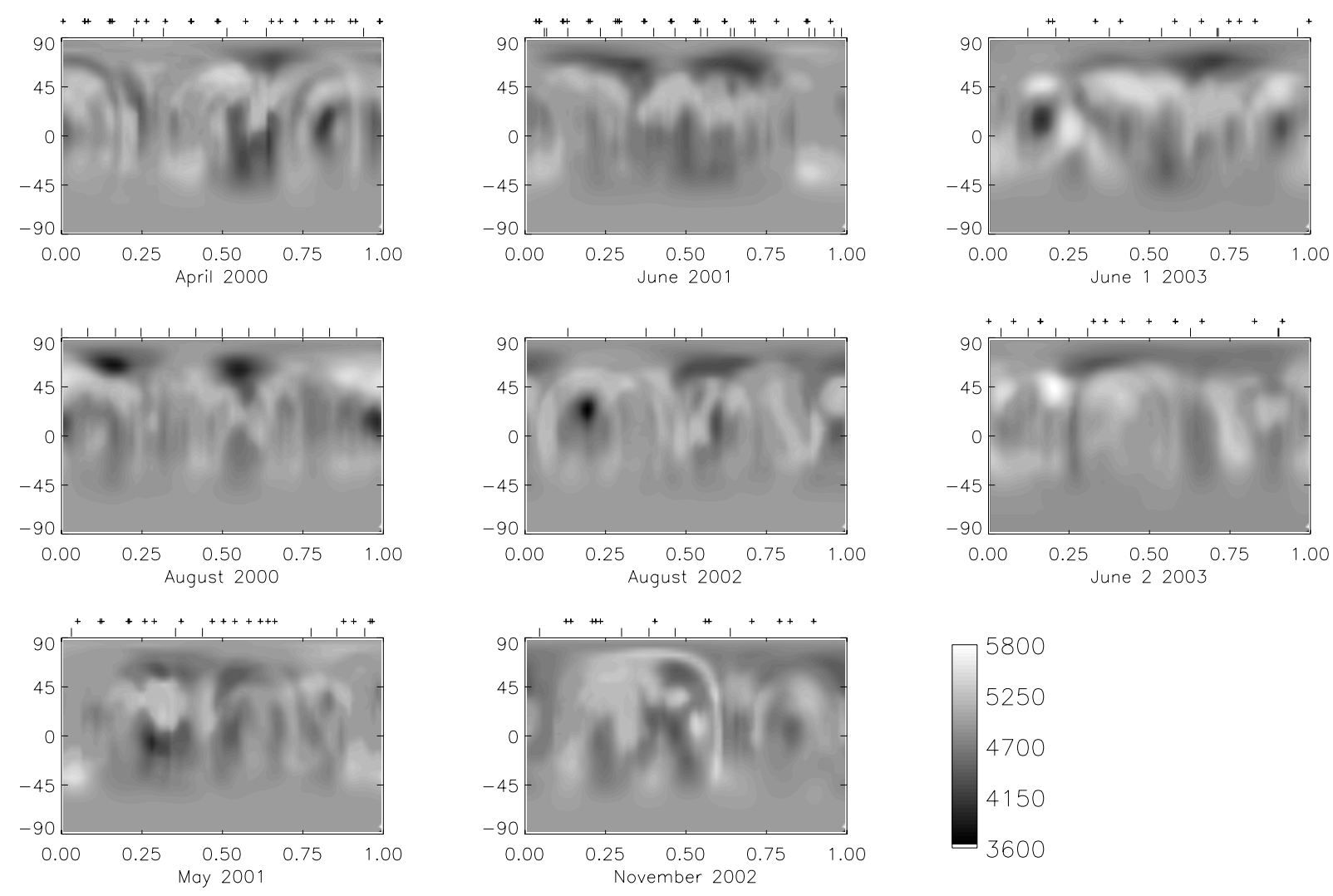

Fig. 1. continued.

and in Korhonen et al. (2001b), photometry was not used as a constraint.

\subsection{Reliability of the maps}

The Doppler maps in Fig. 1 reveal mainly high latitude spots on FK Com. Sometimes spots appear close to the equator or even at the equator. However, many of the maps showing equatorial spots (e.g., June 1993, April 2000, May 2001 and November 2002) are maps for which there are only a few phases in the spectroscopic data set. In such cases the Doppler imaging procedure may not be able to determine the spot latitude and will tend to place the spot near to the centre of the visible stellar disk (see, e.g., Hackman 2004). In the subsequent analysis only the maps where the spot positions were considered reliable were used. The maps with low number of phases $(\leq 5)$ or large phase gaps $(\geq 0.25$ in phase) in the spectroscopic observations were excluded. All photometric observation sets have a good phase coverage.

As discussed earlier, the June 1993 map had a relatively good phase coverage, but the exposure times were too long (up to $120 \mathrm{~min}$ ), and thus the map could be affected by phase smearing and could not be considered reliable. Also, the March 1994 map was excluded from the analysis due to the fact that no spots with temperature less than $4500 \mathrm{~K}$ were seen in it. This map had a very dense data sampling (both in spectra and photometry) and a good $S / N$, but still the coolest areas in the map are only approximately $400 \mathrm{~K}$ below the temperature of the unspotted surface. The reason for the coolest areas being relatively hot could be instrumental (strong interference patterns were seen in some spectra, which have been excluded from the Doppler imaging), or it could be caused by the map's proximity to the photometrically detected flip-flop event in 1994.

The spectroscopic observations for the August 2000 and August 2002 maps were of good quality, but no simultaneous photometric observations were available to verify the spot structures seen in the maps. Furthermore, for both of these maps the light-curve calculated from the map corresponded poorly with the closest photometric observations. The August 2000 map is concluded to be reliable as it most probably reveals rapid spot evolution that occurred in the latter part of the year 2000. This issue is further discussed in Sect. 4.3.

In Fig. 4 the light-curve calculated from the August 2002 map is plotted together with the observed light-curves from June 2002 and December 2002. As can be seen, the light-curve calculated from the temperature map shows a minimum around phases 0.6-0.9. This minimum is seen in both observed light-curves, but stronger than in the calculated one. The second minimum seen in the calculated light-curve around the phases $0.0-0.3$ is not seen in the observations. On the contrary, both observed lightcurves show a maximum around these phases. This could imply that the spots around phases $0.0-0.3$ have emerged since the June photometric observations, and disappeared again before the December observations. Still, the existence of this active region cannot be confirmed with the current data, especially because it is located in a phase gap $(0.13-0.38)$ in the spectroscopic observations. Actually both active regions seen in the August 2002 map are located in relatively large phase gaps (0.25 in phase). For these reasons the reliability of the August 2002 map could not be confirmed, and it was excluded from further analysis.

The maps that were considered reliable and used in the following analysis are denoted " $\mathrm{R}$ " in Table 5. 
Table 5. Summary of the new Doppler images of FK Com. The table gives the location of the largest phase gap, number of phases, the deviation between the observations and the calculated spectra and the corresponding $S / N$, the average $S / N$ of the observations, the temperature range in the map, the phases of areas cooler than the limiting temperature (notations by the spot phases indicate a range of latitudes: $h=45^{\circ}-75^{\circ}, l=20^{\circ}-45^{\circ}$, $e<20^{\circ}, s=$ southern hemisphere) and comments: $R=$ the map was considered reliable both in the spot latitudes and longitudes, and was used in the following analysis, $N=$ no simultaneous photometry.

\begin{tabular}{|c|c|c|c|c|c|c|c|}
\hline Map & Phase Gap & Phases & Dev. $(S / N)$ & $S / N$ & Temperature & Spot phases & $\overline{\text { Remark }}$ \\
\hline June 1993 & $0.43-0.64$ & 7 & $0.724 \%(138)$ & 187 & $4042-5813 \mathrm{~K}$ & $0.18-0.24,0.83-0.88(\mathrm{e}), 0.93-1.06(\mathrm{~h}+\mathrm{e})$ & \\
\hline March 1994 & $0.78-0.97$ & 75 & $0.524 \%(191)$ & 258 & $4627-5436 \mathrm{~K}$ & - & \\
\hline March 1998 & $0.72-0.91$ & 12 & $0.569 \%(176)$ & 184 & $4439-5351 \mathrm{~K}$ & $0.36-0.46(\mathrm{~h})$ & $R$ \\
\hline April 1998_1 & $0.95-0.13$ & 9 & $0.346 \%(289)$ & 344 & $4288-5355 \mathrm{~K}$ & $0.34-0.52(\mathrm{~h}), 0.78-1.0(\mathrm{~h}), 0.95(\mathrm{~s})$ & $R$ \\
\hline April 1998_2 & $0.46-0.64$ & 10 & $0.340 \%(294)$ & 374 & $4472-5311 \mathrm{~K}$ & $0.92-0.02(\mathrm{~h})$ & $R$ \\
\hline July 1998 & $0.14-0.30$ & 13 & $0.428 \%(234)$ & 297 & $4499-5398 \mathrm{~K}$ & $0.28(\mathrm{~h})$ & $R$ \\
\hline April 1999 & $0.29-0.58$ & 17 & $0.853 \%(117)$ & 154 & $4499-5580 \mathrm{~K}$ & $0.30(\mathrm{~h})$ & \\
\hline May 1999 & $0.95-0.03$ & 20 & $0.559 \%(179)$ & 181 & $3804-5580 \mathrm{~K}$ & polar spot, $0.6(\mathrm{~h}), 1.0(\mathrm{~h}), 0.97(\mathrm{~s})$ & $R$ \\
\hline July 1999 & $0.02-0.13$ & 12 & $0.656 \%(152)$ & 215 & $3737-5494 \mathrm{~K}$ & $0.52-0.58(\mathrm{~h}), 0.78-0.98(\mathrm{~h}), 0.91(\mathrm{~s})$ & $R$ \\
\hline March 2000 & $0.95-0.06$ & 20 & $0.421 \%(238)$ & 298 & $4059-5321 \mathrm{~K}$ & $0.50-0.64(\mathrm{~h})$ & $R$ \\
\hline April 2000 & $0.64-0.94$ & 5 & $0.783 \%(128)$ & 152 & $4115-5501 \mathrm{~K}$ & $\begin{array}{l}0.23(\mathrm{l}), 0.52-0.66(\mathrm{e}+\mathrm{s}) \\
0.59-0.70(\mathrm{~h}), 0.78-0.84(\mathrm{e}), 0.97(\mathrm{e})\end{array}$ & \\
\hline August 2000 & $0.25-0.33$ & 12 & $0.564 \%(177)$ & 240 & $3835-5614 \mathrm{~K}$ & $0.06-0.21$ (h), 0.48-0.61 (h), 0.94-1.02 (e) & $R, N$ \\
\hline May 2001 & $0.03-0.35$ & 6 & $0.507 \%(197)$ & 259 & $4014-5599 \mathrm{~K}$ & $0.44-0.78(\mathrm{e}+\mathrm{h}), 0.50-0.56(\mathrm{e}+\mathrm{h})$ & \\
\hline June 2001 & $0.13-0.23$ & 17 & $0.436 \%(229)$ & 316 & $4215-5505 \mathrm{~K}$ & $0.20-0.38(\mathrm{~h}), 0.54-0.74(\mathrm{~h}), 0.49$ (e) & $R$ \\
\hline August 2002 & $\begin{array}{l}0.13-0.38 \\
0.55-0.80\end{array}$ & 7 & $0.473 \%(211)$ & 207 & $3691-5388 \mathrm{~K}$ & $\begin{array}{l}0.16-0.22(\mathrm{l}+\mathrm{e}), 0.48-0.68(\mathrm{~h}) \\
0.60(\mathrm{e}), 0.97(\mathrm{e}), 1.02(\mathrm{~h})\end{array}$ & $N$ \\
\hline November 2002 & $0.64-0.05$ & 5 & $0.708 \%(141)$ & 199 & $4397-5502 \mathrm{~K}$ & $0.44-0.48(\mathrm{~h}+\mathrm{e}), 0.94-1.0(\mathrm{~h})$ & \\
\hline June 2003_1 & $0.71-0.95$ & 8 & $0.395 \%(253)$ & 364 & $4103-5663 \mathrm{~K}$ & $\begin{array}{l}0.13-0.19(\mathrm{e}), 0.56(\mathrm{~s}), 0.59-0.79(\mathrm{~h}) \\
0.89-0.94(\mathrm{e})\end{array}$ & $R$ \\
\hline June 2003_2 & $0.31-0.63$ & 7 & $0.425 \%(235)$ & 336 & $4421-5861 \mathrm{~K}$ & $0.29-0.40(\mathrm{~h})$ & \\
\hline
\end{tabular}

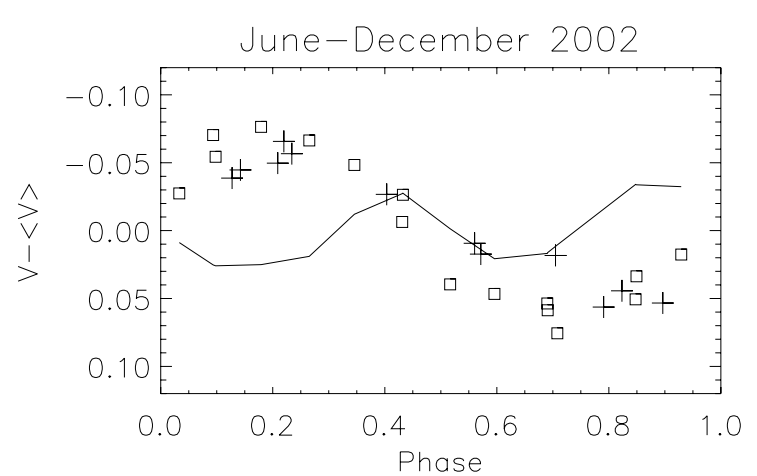

Fig. 4. The $V$ band light-curve for June 2002 (squares), photometry calculated from the August 2002 Doppler image (line) and the $V$ band light-curve for December 2002 (crosses).

\section{Spot properties}

\subsection{Spot latitudes}

The spot latitudes were measured from the Doppler images using the limiting spot temperature of $T_{\lim }=4500 \mathrm{~K}$. Table 6 gives the range of latitudes in the reliable Doppler images. As can be seen, in most cases the spots are located only at high latitudes, i.e., between $47^{\circ}$ and $78^{\circ}$. In some cases the spots also occur closer to the equator, but only three maps, August 2000, June 2001 and June 2003, have spots that are at the equator. In the case of the June 2001 map the equatorial spot is so small that it only occupies a few pixels. The spots usually occupy only one latitude range, but in the three aforementioned maps and in the June 1996 map there are two latitude ranges that are active. For young rapidly rotating solar-like stars such a bimodal distribution of spot latitudes has been suggested by thin flux tube models (see, e.g., Schüssler et al. 1996; Granzer et al. 2000).
To calculate the average spot latitude, the areas cooler than $T_{\lim }$ at individual latitude strips were measured. The areas of the spots at each latitude were used as weights. In the calculation of the average latitude the few cool features seen in the "southern" hemisphere were excluded. This was done because the Doppler imaging methods cannot reliably recover spots on the less visible stellar hemisphere. The average spot latitudes calculated from the Doppler images that were considered reliable varied between $32^{\circ} .0 \pm 4^{\circ} .6$ and $74^{\circ} .0 \pm 0.6$, the average of all measurements being $59^{\circ} .9$ and the median $63^{\circ} .6$. The errors were calculated as the error of the weighted average. The average spot latitudes for all the reliable Doppler images are given in Table 6.

\subsection{Active longitudes}

Long term photometric observations of FK Com show that the spots tend to follow two migrating permanent active longitudes that are $180^{\circ}$ apart (Korhonen et al. 2002, 2004). In Fig. 5 the spots determined from $V$ band light-curves (taken from Korhonen et al. 2004) and the spots determined from the Doppler images presented in this paper are plotted against the Julian Date. Note that in both cases, only the main active regions are shown in the plot. For the Doppler images the mean phase of the largest area with a temperature less than $T_{\text {lim }}=4500 \mathrm{~K}$ is used (phases given in Table 5). For cases where there are two close-by spots in the Doppler image (separated by 0.2 in phase or less), the average longitude of these two spots is used. The August 1994 and April 1997 maps have one very wide active region, at phases $0.8-1.3$ and $0.6-1.1$, respectively. In these two cases the average of all the spots is used as the spot phase. In the July 1995 and May 1999 maps there is almost a polar spotlike structure with two appendages towards the lower latitudes. For the July 1995 map the main spot phase is determined as the phase of the larger appendage, and for the May 1999 map both appendages are given as they are of same size. The circles in the plot are spot locations determined from the photometry and 
Table 6. Spot latitudes on each reliable map for the year 1993-2003. The name of the map, the average Heliocentric Julian Date, the spot latitudes on the northern hemisphere and the average spot latitude are given.

\begin{tabular}{|c|c|c|c|}
\hline Map & $\begin{array}{l}\text { HJD } \\
\end{array}$ & latitude & 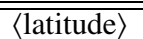 \\
\hline August 1994 & 49583.9 & $47^{\circ}-74^{\circ}$ & $61^{\circ} 2 \pm 1.1$ \\
\hline July 1995 & 49916.0 & $47^{\circ}-78^{\circ}$ & $65^{\circ} .2 \pm 1.5$ \\
\hline June 1996 & 50266.3 & $25^{\circ}, 43^{\circ}-61^{\circ}$ & $48^{\circ} .2 \pm 1.5$ \\
\hline April 1997 & 50550.3 & $56^{\circ}-74^{\circ}$ & $63.6 \pm 0.9$ \\
\hline June 1997 & 50622.4 & $38^{\circ}-70^{\circ}$ & $56^{\circ} .2 \pm 1.4$ \\
\hline January 1998 & 50820.5 & $47^{\circ}-56^{\circ}$ & $53^{\circ} 0 \pm 0.5$ \\
\hline March 1998 & 5088 & $65^{\circ}-70^{\circ}$ & $67^{\circ} .5 \pm 0.4$ \\
\hline April_1 1998 & 50904.4 & $70^{\circ}-79^{\circ}$ & $74^{\circ} 0 \pm 0.6$ \\
\hline April_2 1998 & 50920.4 & $74^{\circ}$ & $74^{\circ, *}$ \\
\hline July $\overline{1998}$ & 51004.9 & $70^{\circ}$ & $70^{\circ, *}$ \\
\hline May 1999 & 51328.5 & $56^{\circ}-79^{\circ}$ & $67^{\circ} .2 \pm 1.2$ \\
\hline July 1999 & 51388.9 & $47^{\circ}-74^{\circ}$ & $61^{\circ} .5 \pm 1.2$ \\
\hline March 2000 & 51651.3 & $61^{\circ}-74^{\circ}$ & $66^{\circ} .4 \pm 0.8$ \\
\hline Augu & 51769.9 & $\begin{array}{c}2^{\circ}-25^{\circ} \\
38^{\circ}-70^{\circ}\end{array}$ & $38^{\circ} .2 \pm 4^{\circ} .4$ \\
\hline June 2001 & 52068.4 & $0^{\circ}, 52^{\circ}-74^{\circ}$ & $60^{\circ} .2 \pm 2.6$ \\
\hline June 2003_1 & 52797.9 & $\begin{array}{c}2^{\circ}-25^{\circ} \\
56^{\circ}-74^{\circ}\end{array}$ & $32^{\circ} .0 \pm 4^{\circ} .6$ \\
\hline
\end{tabular}

* All the spots on these maps were on the same latitude.

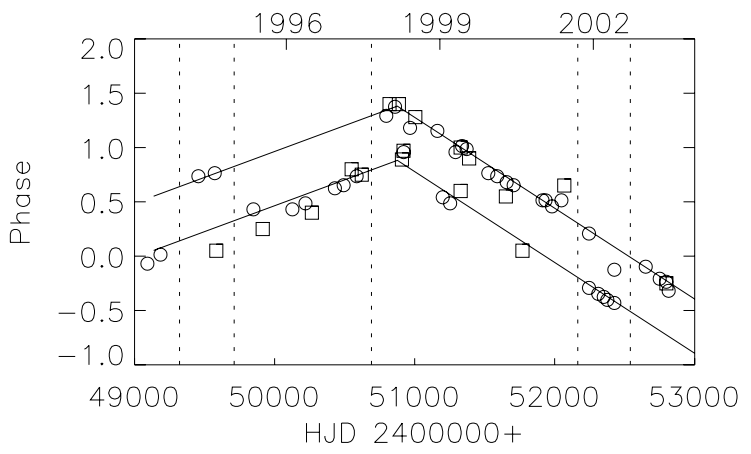

Fig. 5. Active longitudes on FK Com for the years 1994-2003. The phases of the main spots from photometry (circles) and from Doppler images (squares) are plotted against the Julian Date. Solid lines give the active longitudes determined from photometry and the dashed lines denote the times of the flip-flop events. The photometric spot phases and the active longitudes are taken from Korhonen et al. (2004).

the squares are from the temperature maps. The solid lines give the active longitudes determined from the photometry and the dashed lines are the locations of the flip-flop events, also based on photometry.

The main spots determined from the Doppler images coincide reasonably well with the active longitudes based on the photometry. To illustrate this further, the fractional spot coverage at each rotational phase is calculated for three maps (June 1997, April_1 1998 and July 1999), and compared with the phases of the photometrically determined active longitudes for these epochs. In Fig. 6 the fractional spot coverage on the "northern" hemisphere (the area at each longitude that has a temperature less than $T_{\lim }=4500 \mathrm{~K}$ ) is given with a solid line, and the locations of the photometrically determined active longitudes by dashed lines. As can be seen, the spots on the Doppler images are located close to the earlier detected active longitudes. Especially the main spots correlate well with the active longitudes.
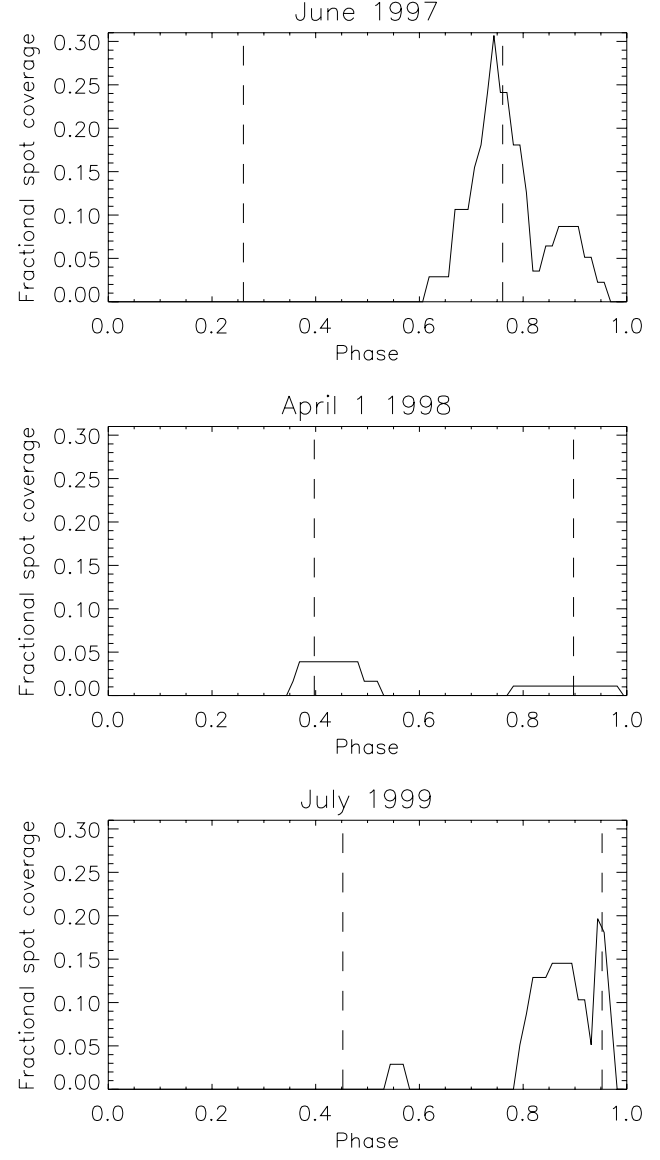

Fig. 6. The fractional spot coverage at each rotational phase determined from the June 1997, April_1 1998 and July 1999 maps. The solid line gives the spot coverage in the "northern" hemisphere at each rotational phase and the dashed lines mark the phases of the photometrically determined active longitudes for each epoch.

The active regions measured from the Doppler images for 1994-1996 consistently have a slightly smaller phase than that measured from the photometry (see Fig. 5). Also, the spot phase determined from the August 1994 temperature map does not confirm the main spot phase based on the photometry. This could be due to the map being close in time to the flip-flop event occurring in late 1994. The main active regions determined from the Doppler images confirm the existence of two permanent migrating active longitudes on FK Com, which were earlier detected using long term photometric observations.

\subsection{Short term spot evolution}

In most cases the time difference between consecutive temperature maps is too long to study the short term spot changes. Still, there are several interesting time periods where this investigation can be carried out. These cases are discussed in more detail in the following.

\subsubsection{The years 1998-1999}

There are five reliable maps from the first half of 1998 and two from 1999. In 1998 the spots concentrate around the phases 0.0 and 0.4 and in 1999 around the phases 0.0 and 0.6. This is consistent with the behaviour seen in the long term photometry by Oláh et al. (2006). They report that near the Julian Date 


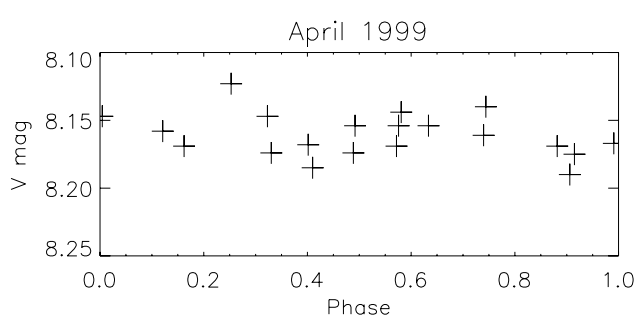

May 1999
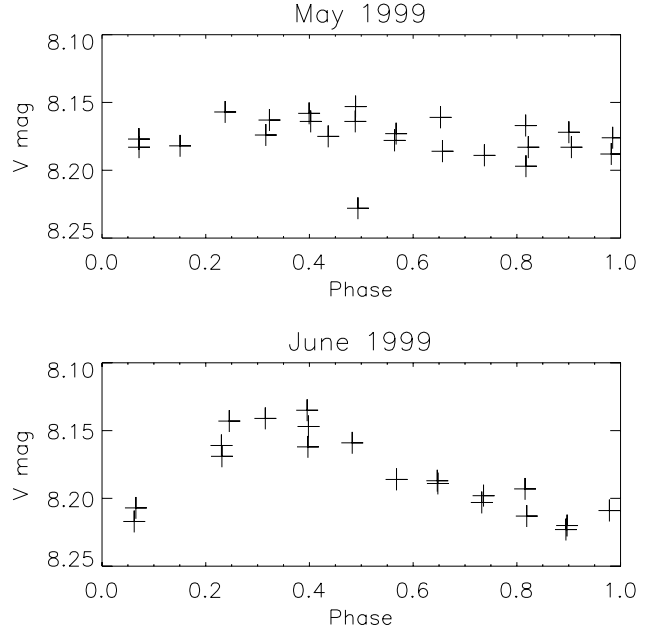

Fig. 7. The $V$ band light-curves for April, May and June 1999.

2451300 (May 1, 1999) the sizes of the two spots seen on the surface around the phases 0.0 and 0.45 gradually decrease and finally the spots almost vanish. Thereafter two new spots appear on the surface around phases 0.0 and 0.70. Our May 1999 map is from the same time period as the detailed investigation presented in Oláh et al. (2006). This map shows similar behaviour as seen in the photometry, i.e., two spots of equal size, one around phase 0.0 and the other one around 0.6 (from Doppler imaging) or 0.7 (from photometry). The other Doppler images are either before or after the dates that were used for the detailed investigation by Oláh et al. (2006) and as such no direct comparison of the results can be carried out. Note that for these temperature maps the photometry was used as a constraint, so one would expect that the maps reflect the same spot evolution as seen in the light-curves.

Between January 1998 and April 1999 the light-curve of FK Com is relatively flat, but as can be seen in Fig. 7 between May 1999 and June 1999 a change in the amplitude occurs. In May 1999 the full amplitude of the $V$ light-curve is 0.09 , and in June $19990^{\mathrm{m}}$. 18. From the temperature maps it can been seen that the spots become more concentrated. The change already begins between April and May 1999, but it is most noticeable, and seen especially in the light-curves between May and June 1999.

\subsubsection{August 2000}

The quality of the August 2000 spectroscopic observations was very good and thus it was puzzling to see the poor fit between the observed $V$ light-curve from June-July 2000 and the one calculated from the map (see Fig. 8a). To explain this we investigated the light-curve evolution of FK Com during the latter part of 2000 and noticed that there were significant changes in the a) August 2000 , original
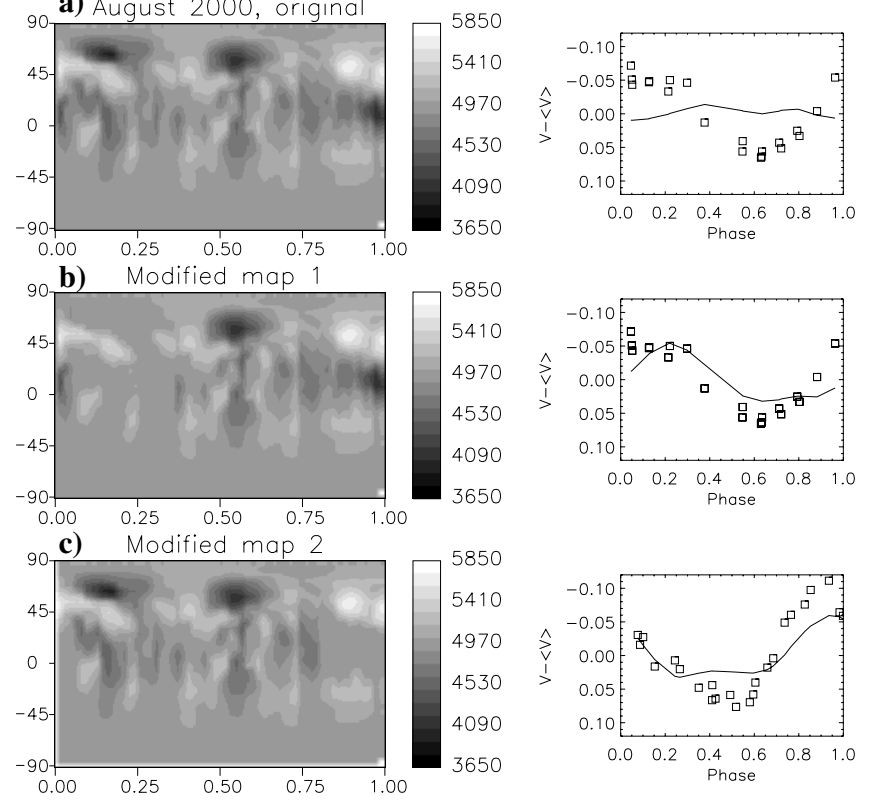

Fig. 8. Rapid spot changes in June-August 2000. a) The August 2000 temperature map and the June-July light-curve (squares) together with the light-curve calculated from the map (solid line). b) The modified map 1: the August 2000 map from which the spots around phase 0.2 have been removed. The light-curve calculated from the map is compared with the June-July light-curve. c) The modified map 2: the August 2000 map from which the spots around phase 1.0 have been removed. The light-curve calculated from the map is compared with the December 2000 light-curve.

light-curves, although the spot locations were similar to those in March and April 2000.

To check whether the August 2000 map could be a transitory stage between the June-July and December spot distributions, some spots were artificially removed from the temperature map and the light-curves from these modified maps were calculated. The results are shown in Fig. 8. The modified map 1 (spots at the phase 0.2 have been removed, Fig. 8b) correlates well with the observed June-July light-curve. The modified map 2 (spots removed at the phase 1.0, Fig. 8c) gives a light-curve similar to the one in December 2000. This suggests that the active region at the phase 1.0 disappeared during July-August 2000 while a new active region was formed at the phase 0.2 . The correlation between the light-curves calculated from the modified map 2 and observed in December 2000 is not perfect. This suggests that some spot evolution happened during the four month period between the spectroscopic observations and the December 2000 light-curve. The light-curves calculated from the modified map 1 fit the June-July photometry very well.

\subsubsection{June 2003}

The spectra from June 2003 span 20 days. When investigating these spectra in detail, it was noted that the line profile shapes of the close-by phases which were observed at different times were not the same. For this reason it was decided to use the spectra to construct two separate temperature maps. In the temperature map from the early part of June 2003 (see Fig. 1 and Table 5) the most prominent spot is located close to the equator around the phase 0.15 . Two other spots, at high latitudes spanning phases $0.6-0.8$ and close to the equator around phase 0.9 , were also 


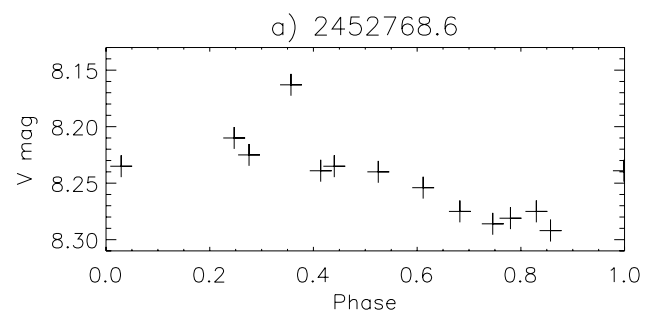

b) 2452794.3
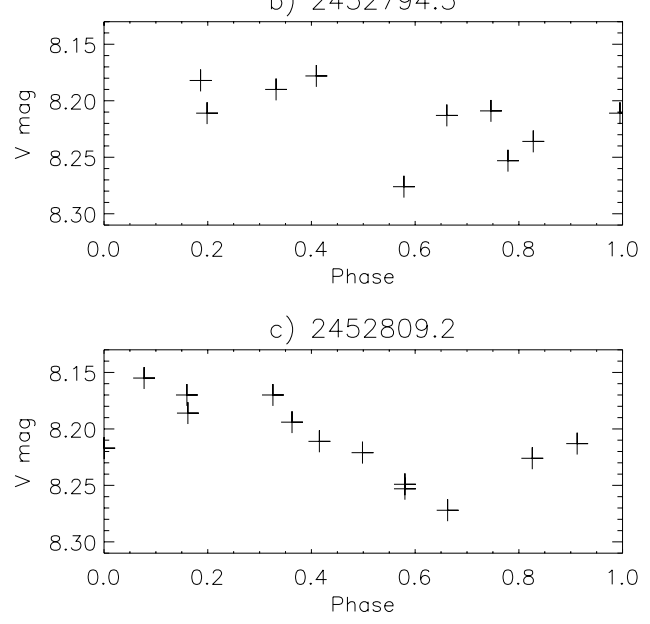

Fig. 9. The $V$ band light-curves in 2003: a) centred at 9th of May 2003, b) centred at 3rd of June 2003 and c) centred at 18th of June 2003.

seen. For the latter June 2003 map, only one spot with temperatures cooler than the limiting temperature, $T_{\mathrm{lim}}$, is seen. This spot is located at high latitudes around phases $0.3-0.4$. Another set of spots, spanning a wide range of latitudes, can be seen around the phases $0.6-0.9$, but these spots have a low contrast. It seems that the whole spot configuration changed within a very short time. However, the observations for the latter June 2003 map had a poor phase coverage, especially between the phases 0.3 and 0.9 .

The changes in the spot configuration on FK Com during June 2003 can also be seen in the light-curves. In Fig. 9 the $V$ band light-curves for three epochs are shown: a) centred at 9th of May 2003, b) centred at 3rd of June 2003 and c) centred at 18th of June 2003. The light-curve from May 2003 shows a minimum around the phases $0.8-1.0$. In early June the whole light-curve is more noisy and does not show a clear minimum. In late June 2003 a minimum appears around the phases $0.6-0.8$, and there are no indications of spots at the phase 0.2 . The lightcurves support the interpretation obtained from the Doppler images, i.e., that the spot seen at the phase 0.2 in early June disappears very rapidly in mid-June. The location of the minimum has also changed between May and June, implying that also other changes in the spot configuration have occurred.

\section{Differential rotation}

Assuming that the differential rotation law of the Sun can be generalised to stars, the surface rotation can be expressed by

$\Omega=\Omega_{\mathrm{eq}}+\beta \sin ^{2} \psi$,

where $\psi$ is the latitude, $\Omega_{\mathrm{eq}}$ is the equatorial angular velocity and $\beta$ defines the magnitude of the differential rotation. The relative differential rotation coefficient is given by

$\alpha=\frac{\Omega_{\mathrm{eq}}-\Omega_{\mathrm{pol}}}{\Omega_{\mathrm{eq}}}$ or $\alpha=\frac{-\beta}{\Omega_{\mathrm{eq}}}$
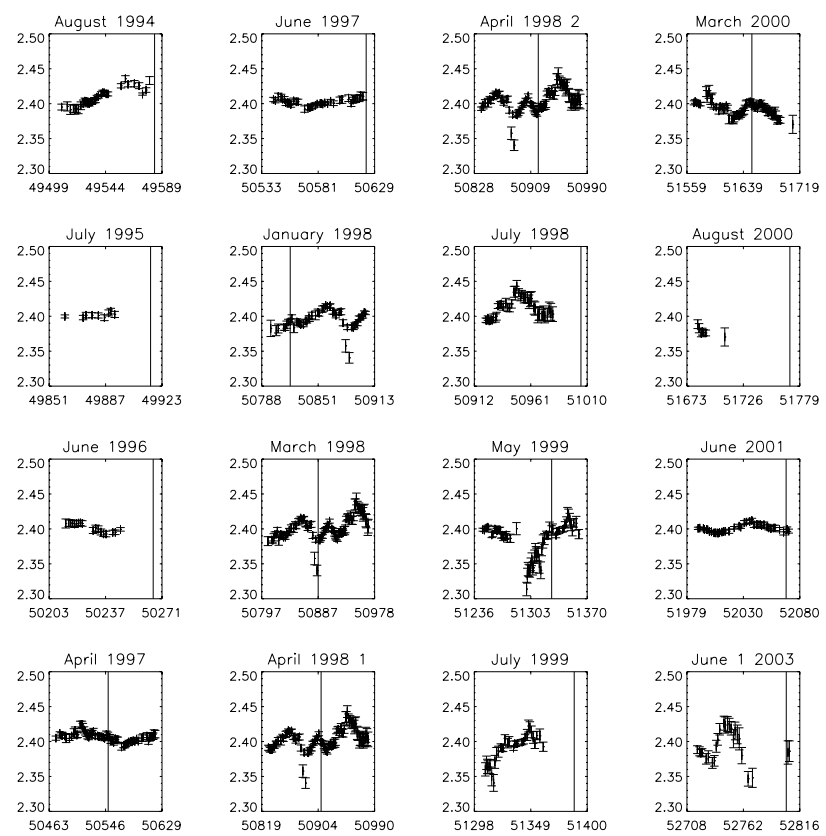

Fig. 10. The results from the period determination for the epochs of reliable Doppler maps. In each plot the abscissa is the Julian Date and the ordinate the period in days. Each mark denotes a reliable period measurement, the height of the mark corresponding to the error. The vertical lines shows the epochs of the Doppler maps.

where $\Omega_{\mathrm{pol}}$ is the polar angular velocity. Therefore, to measure the surface differential rotation in stars, estimates of the spot latitude and the spot rotation period are needed.

The value of differential rotation for FK Com was estimated by combining the information of the spot latitudes from Doppler images and periods from the photometric observations. The photometric rotation period at, or close to, the epoch of the Doppler images was determined by applying the "three stage period analysis" (TSPA, Jetsu \& Pelt 1999) to the $V$ band observations. In the investigation, subsets of photometric data corresponding to each Doppler map were created by selecting all data points 100 days before and after each map, when available. The spot rotation period was determined using data from 35 days. Then the subset was moved by 1 data point (but at least by 0.5 days) and a new determination was done. The whole subset for each map was analysed this way.

This way we obtained 7 to 92 periods around each Doppler map (the average being 47). In Fig. 10 the results from the period determination are shown for the epochs of the reliable Doppler maps. In the plot the time of the Doppler map is given by a vertical line. As can be seen from the figure, in some years the spot rotation period can change significantly on short time scales (like during 1998) and sometimes it is relatively stable (e.g., 1997). We emphasise that these periods were not independent measurements, since neighbouring points were derived from nearly the same data.

The spot rotation period, corresponding to each Doppler map, was retrieved by averaging the 10 reliable period measurements closest in time to each map. The reliability of the periods were estimated by using the same rejection criteria as in Jetsu $\&$ Pelt (1999). Due to lack of data only 9 reliable periods were determined within the chosen time period for the July 1995 map and 7 for the August 2000 map. Also, in the case of June 2003 only 2 reliable periods closest to the map were used. This was 
due to the lack of reliable periods and large variations in the period values during this subset, however the two reliable periods that coincide with the Doppler map give identical values and can be considered reliable. The errors of the periods were determined using the bootstrap method, and are also plotted in Fig. 10. The inverse square of the error was used as the weight when determining the period for the epoch of the Doppler image.

The period changes were so rapid in March-April 1998 that averaging the 10 measurements was meaningless. Furthermore, the light-curve for this epoch is almost flat (see Korhonen et al. 2002) and rapid changes in the light-curve occur, implying that the period changes seen around this time could be caused by changes in the spot configuration and not in the spot rotation period itself. Also, for July 1998 the Doppler map is relatively far apart in time from the photometric observations and the period is highly variable, so it is not meaningful to determine the period for this epoch. The period determination for the August 2000 map is not taken into account when measuring the differential rotation, as there is a time gap of more than one month between the spectroscopic and photometric observations, and significant spot changes have occurred during that time (see Fig. 8). For other maps, where the time of the Doppler image did not coincide with that of the period determination, the behaviour of the spot rotation period is much more stable and these epochs were used to determine the surface differential rotation.

The angular velocities were calculated from the spot rotation periods for the maps in 1995-1997 and 1999-2003. The values are plotted versus the $\sin ^{2} \psi$ in Fig. 11 together with the errors. The latitude errors are from Table 6 and period errors are determined by taking the standard deviation of the measurements used in obtaining the average period and divided by the square-root of the number of data points. Here $\psi$ is the average spot latitude (see Sect. 4.1). A line was fitted to the data points using Chi-square minimisation. The errors of the angular velocities and latitudes were used in the minimisation. From the fit, $\beta$ and the equatorial angular velocity $\Omega_{\mathrm{eq}}$ can be obtained. The fit yields $\beta=-1.78^{\circ} /$ day $\pm 0.12^{\circ} /$ day and $\Omega_{\mathrm{eq}}=$ $151.30^{\circ} /$ day $\pm 0.09^{\circ} /$ day. These values together with Eq. (2) give $\alpha=0.012 \pm 0.002$. This value implies solar-type surface differential rotation on FK Com, with somewhat smaller $\beta$ than observed on the Sun $(\beta=-2.9$, Balthasar et al. 1986) and with a much smaller relative differential rotation coefficient. However, the scatter in the data points around the fitted curve implies that our result should be interpreted more as an estimate of the magnitude of the differential rotation than an exact solution of the differential rotation curve.

Photometry is more affected by low latitude spots. In the cases where both low and high latitude spots co-exist, it is possible that the lower latitude spots will dominate the photometric period, even though the higher latitude spots could be larger and influence more the average spot latitude. In such a case our analysis would be biased leading to an underestimation of the surface differential rotation.

\section{Discussion}

\subsection{Spot lifetimes}

Earlier studies based on photometric observations showed spots on FK Com grouping at two permanent active longitudes (Jetsu et al. 1993; Korhonen et al. 2002). This behaviour is also seen in our Doppler images (see Fig. 5). The same active region can be followed for at least three years, e.g., in 1995-1997. The dominant of these active regions often shows two main spots,

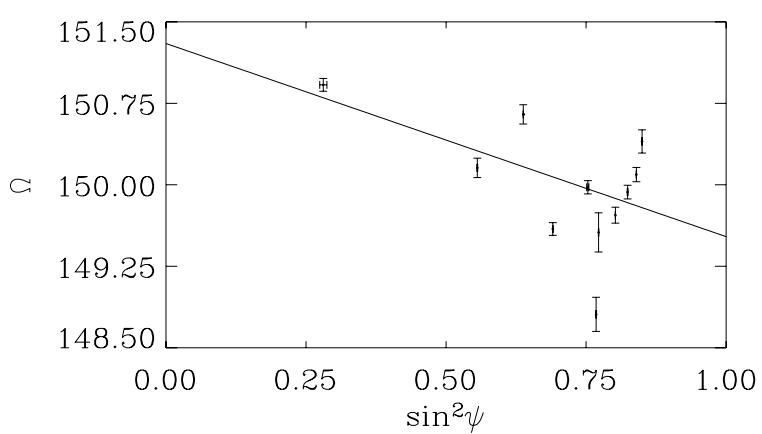

Fig. 11. Angular velocities, $\Omega$, in degrees per day from the period measurements from the long term photometry versus the $\sin ^{2}$ of the average spot latitude in Doppler maps of the same epoch. The errors in latitude and angular velocities are marked. The solid line is the fit to the measurements.

although the precise configuration changes from one Doppler image to another. This implies that the individual spots appear and disappear within the active region during the three years that the active region itself exists, and the spot lifetime seems to be months instead of years. Of course, some of the changes between the maps can be due to inaccuracies in the maps themselves.

As discussed in Sect. 4.3, for some epochs e.g., May-June 1999 and May-June 2003, the spot evolution is very rapid and rearrangements of the spots can happen within days. Also, the spot rotation period sometimes, especially during the year 1998 , shows rapid variations that are most likely due to spot configuration changes. On the whole, it can be concluded that the active regions on FK Com persist for years, but the exact spot configuration within these regions changes on much shorter time scales, sometimes within days.

Based on numerical simulations of bipolar magnetic regions, Işık et al. (2007) have shown that in a rapidly rotating active star of solar radius the expected spot lifetime is a few months. The exact lifetime depends on the spot latitude (mid-latitude spots live a shorter time than equatorial or high latitude spots) and spot size (larger spots live longer). The simulations for sub-giants show even longer spot lifetimes. For a spot at latitude $60^{\circ}$ the lifetime on a sub-giant is 9-10 months (instead of 55 days for a star with a solar radius) and higher latitude spots can even live years, explaining the stable polar spots seen in many active giants and sub-giants.

The radius of FK Com $\left(R \approx 8 R_{\odot}\right)$ is larger than the one used in the simulations for sub-giants $\left(3.3 R_{\odot}\right)$ by Işı k et al. (2007), but it is still interesting to compare the results obtained in this study to the ones obtained by simulations. Most of the spots on FK Com are at high latitudes, the average spot latitude of all the maps being 59.9. This would imply a spot lifetime of 910 months. The temperature maps for some epochs confirm this behaviour. For example, the July 1999 and March 2000 maps have very similar spot configurations, only the relative strengths of the spots change, indicating that these spots have existed for at least 8 months on the surface of FK Com. On the other hand, the time period of largest average spot latitude (March-July 1998), which according to simulations should show the most stable spots, shows many very rapid changes in the spot configuration.

\subsection{On the differential rotation of FK Com}

In the earlier studies of FK Com two methods have been used to measure the surface differential rotation. In Paper II 6 consecutive Doppler images spanning 3 years were cross-correlated. 
This study yielded an almost solid body rotation of the stellar surface. In Korhonen et al. (2004) the surface differential rotation was deduced by combining the rotation period of the active longitudes and the latitude information from the Doppler images. This study gave a solar-type differential rotation with $\alpha=0.016 \pm 0.004$, similar to the result obtained in Sect. 5 of this paper. Unfortunately, the latitudes used in that study were not accurate. There are are also two other problems in using this method: 1) An average spot rotation period for a time interval of several years is used, and changes in the spot rotation period seem to occur even on short time scales (see Fig. 10); 2) the average latitudes also change significantly from one Doppler map to another (see Table 6). It does not seem to be meaningful to use a method based on rotation periods and latitudes averaged over a long time interval to determine the surface differential rotation on FK Com.

In this paper the latitude information from the Doppler images and the spot rotation period from photometry is used to determine the surface differential rotation. When using this method one has to be pay attention to the period determination; rapid changes in the spot configuration may affect the period determination. An additional problem is that on FK Com the spots are usually at high latitudes. There is only one data point at low latitudes $\left(<40^{\circ}\right)$. This point has a strong influence on the surface differential rotation determined with this method. If this point is removed, the result will still be a solar-type differential rotation, but with even smaller $\alpha$.

The method using latitude information from the Doppler images and the spot rotation period from the photometry yields solar type differential rotation with a relative differential rotation coefficient $\alpha$ less than $10 \%$ of the solar value and $\beta$ smaller than the solar value. The rotation law of FK Com correlates well with the recent results by Barnes et al. (2005), where they investigate the dependence of $\beta$ on temperature and rotation in 10 young solar type stars. For another FK Com-type star, HD 199178, both solar-type surface differential rotation (Petit et al. 2004) and antisolar rotation (Hackman et al. 2001) have been suggested.

\section{Conclusions}

The investigation of 25 Doppler maps of FK Com yielded the following results:

- The longitudes of the main spots determined from the surface temperature maps follow well the position of the permanent active longitudes that have been determined previously from the long term photometry.

- The same active longitude can be active for several years at a time, but the detailed spot configuration changes from map to map, implying that the spot lifetime is much shorter.

- During some epochs spot changes occur even on time scales of days and weeks.

- Surface differential rotation measurements using latitude information from the Doppler images and period information from the photometric observations yield a surface differential rotation law for FK Com of $\Omega=\left(151.30^{\circ} /\right.$ day $\pm 0.09^{\circ} /$ day $)-$ $\left(1.78^{\circ} /\right.$ day $\pm 0.12^{\circ} /$ day $) \sin ^{2} \psi$ and $\alpha=0.012 \pm 0.002$.

Acknowledgements. The authors would like to thank the anonymous referee for his/her very useful comments that helped to improve this paper considerably. The NAO Rozhen staff is acknowledged for supporting the observations.
The work of H.K. was partly supported by the Finnish graduate school in Astronomy and Space Physics and Vilho, Yrjö and Kalle Väisälä Foundation. H.K. and K.G.S. acknowledge the Deutsche Forschungsgemeinschaft grant KO 2320/1-2. K.G.S. also acknowledges the Deutsche Forschungsgemeinschaft grant STR 645/1. S.V.B. acknowledges the EUYRI Award from the ESF. Nordic Optical Telescope is operated on the island of La Palma jointly by Denmark, Finland, Iceland, Norway, and Sweden, in the Spanish Observatorio del Roque de los Muchachos of the Instituto de Astrofisica de Canarias.

\section{References}

Baliunas, S. L., Horne, J. H., Porter, A., et al. 1985, ApJ, 294, 310 Balthasar, H., Vázquez, M., \& Wöhl, H. 1986, A\&A, 155, 87

Barnes, J. R., Collier Cameron, A., Donati, J.-F., et al. 2005, MNRAS, 357, L1 Bellot Rubio, L. R., Schlichenmaier, R., \& Tritschler, A. 2006, A\&A, 453, 1117 Berdyugina, S. V., \& Tuominen, I. 1998, A\&A, 336, L25

Bopp, B. W., \& Rucinski, S. M. 1981, in Fundamental Problems in the Theory of Stellar Evolution, ed. D. Sugimoto, D. G. Lamb, \& D. N. Schramm (Reidel, Dordrecht), IAU Symp., 93, 177

Chugainov, P. F. 1966, Inf. Bull. Var. Stars, No. 172

Donati, J.-F., \& Collier Cameron, A. 1997, MNRAS, 291, 1

Dorren, J. D., Guinan, E. F., \& McCook, G. P. 1984, PASP, 96, 250

Granzer, Th., Schüssler, M., Caligari, P., \& Strassmeier, K. G. 2000, A\&A, 355, 1087

Hackman, T. 2004, Doppler imaging and photometric time series analysis of stellar spot activity, Academic dissertation, University of Helsinki

Hackman, T., Jetsu, L., \& Tuominen, I. 2001, A\&A, 374, 171

Henry, G. W., Eaton, J.A., Hamer, J., \& Hall, D. S. 1995, ApJS, 97, 513

Huenemoerder, D. P., Ramsey, L. W., Buzasi, D. L., \& Nations, H. L. 1993, ApJ, 404,316

Ilyin, I. V. 2000, High resolution SOFIN CCD échelle spectroscopy, Academic dissertation, University of Oulu

Işık, E., Schüssler, M., \& Solanki, S. K. 2007 A\&A, 464, 1049

Jetsu, L., \& Pelt, J. 1999, A\&AS, 139, 629

Jetsu, L., Pelt, J., Tuominen, I., \& Nations, H. L. 1991, in The Sun and Cool Stars: activity, magnetism, dynamos, ed. I. Tuominen, D. Moss, \& G. Rüdiger (Heidelberg: Springer), Proc. IAU Coll., 130, 381

Jetsu, L., Pelt, J., \& Tuominen, I. 1993, A\&A, 278, 449

Jetsu, L., Tuominen, I., Antov, A., et al. 1994a, A\&AS, 103, 183

Jetsu, L., Tuominen, I., Grankin, K. N., Mel'nikov, S. Yu., \& Shevchenko, V. S. 1994b, A\&A, 282, L9

Kitchatinov, L. L., \& Rüdiger, G. 1999, A\&A, 344, 911

Korhonen, H., Berdyugina, S. V., Hackman, T., et al. 1999, A\&A, 346, 101 (Paper I)

Korhonen, H., Berdyugina, S. V., Hackman, T., Strassmeier, K. G., \& Tuominen, I. 2000, A\&A, 360, 1067 (Paper II)

Korhonen, H., Berdyugina, S. V., Tuominen, I., et al. 2001a, A\&A, 374, 1049

Korhonen, H., Berdyugina, S.V., Strassmeier, K. G., \& Tuominen, I. 2001b, A\&A, 379, L30

Korhonen, H., Berdyugina, S. V., \& Tuominen, I. 2002, A\&A, 390, 179

Korhonen, H., Berdyugina, S. V., \& Tuominen, I. 2004, AN, 325, 402

Lanza, A. F., Catalano, S., Rodonò, M., et al. 2002, A\&A, 386, 583

Merrill, P. W. 1948, PASP, 60, 382

Oláh, K., Korhonen, H., Kővári, Zs., Forgács-Dajka, E., \& Strassmeier, K. G. 2006, A\&A 452, 303

Oliveira, J. M., \& Foing, B. H. 1999, A\&A, 343, 213

Petit, P., Donati, J.-F., \& Collier Cameron, A. 2002, MNRAS, 334, 374

Petit, P., Donati, J.-F., Oliveira, J. M., et al. 2004, MNRAS, 351, 826

Piskunov, N. E., Huenemoerder, D. P., \& Saar, S. H. 1994, in Proceedings of the Eighth Cambridge Workshop on Cool Stars, Stellar Systems, and the Sun, ed. J. P. Caillault, ASPC, 64, 658

Ramsey, L. W., Nations, H. L., \& Barden, S. C. 1981, ApJ, 251, 101

Reiners, A., \& Schmitt, J. H. M. M. 2002, A\&A, 384, 155

Reiners, A., \& Schmitt, J. H. M. M. 2003, A\&A, 398, 647

Rodonò, M., Messina, S., Lanza, A.F., Cutispoto, G., \& Teriaca, L. 2000, A\&A, 358,624

Schüssler, M., Caligari, P., Ferriz-Mas, A., Solanki, S. K., \& Stix, M. 1996, A\&A, 314, 503

Strassmeier, K. G., Bartus, J., Cutispoto, G., \& Rodonó, M. 1997, A\&AS, 125, 11

Vogt, S. S., Hatzes, A. P., Misch, A., \& Kürster, M. 1999, ApJS, 121, 546

Weber, M., \& Strassmeier, K. G. 2001, A\&A, 373, 974

Weber, M., Strassmeier, K. G., \& Washuettl, A. 2005, AN, 326, 287 
H. Korhonen et al.: Study of FK Comae Berenices. V., Online Material p 1

\section{Online Material}


H. Korhonen et al.: Study of FK Comae Berenices. V., Online Material p 2
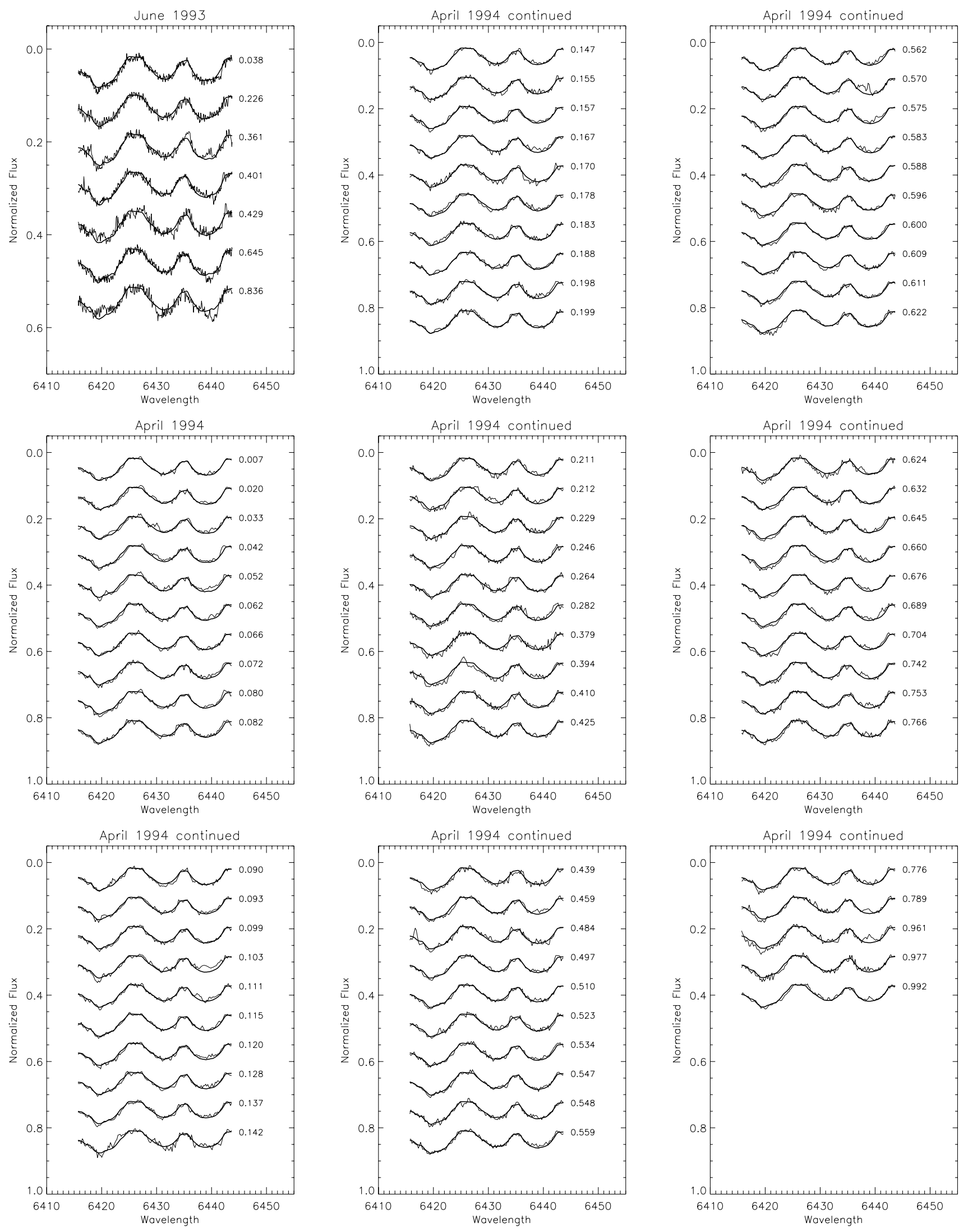

Fig. 2. The spectra used in the Doppler imaging for the years 1993-2003 for the previously unpublished data sets. Calculated and observed spectral lines are shown by thick and thin lines, respectively. 
H. Korhonen et al.: Study of FK Comae Berenices. V., Online Material p 3
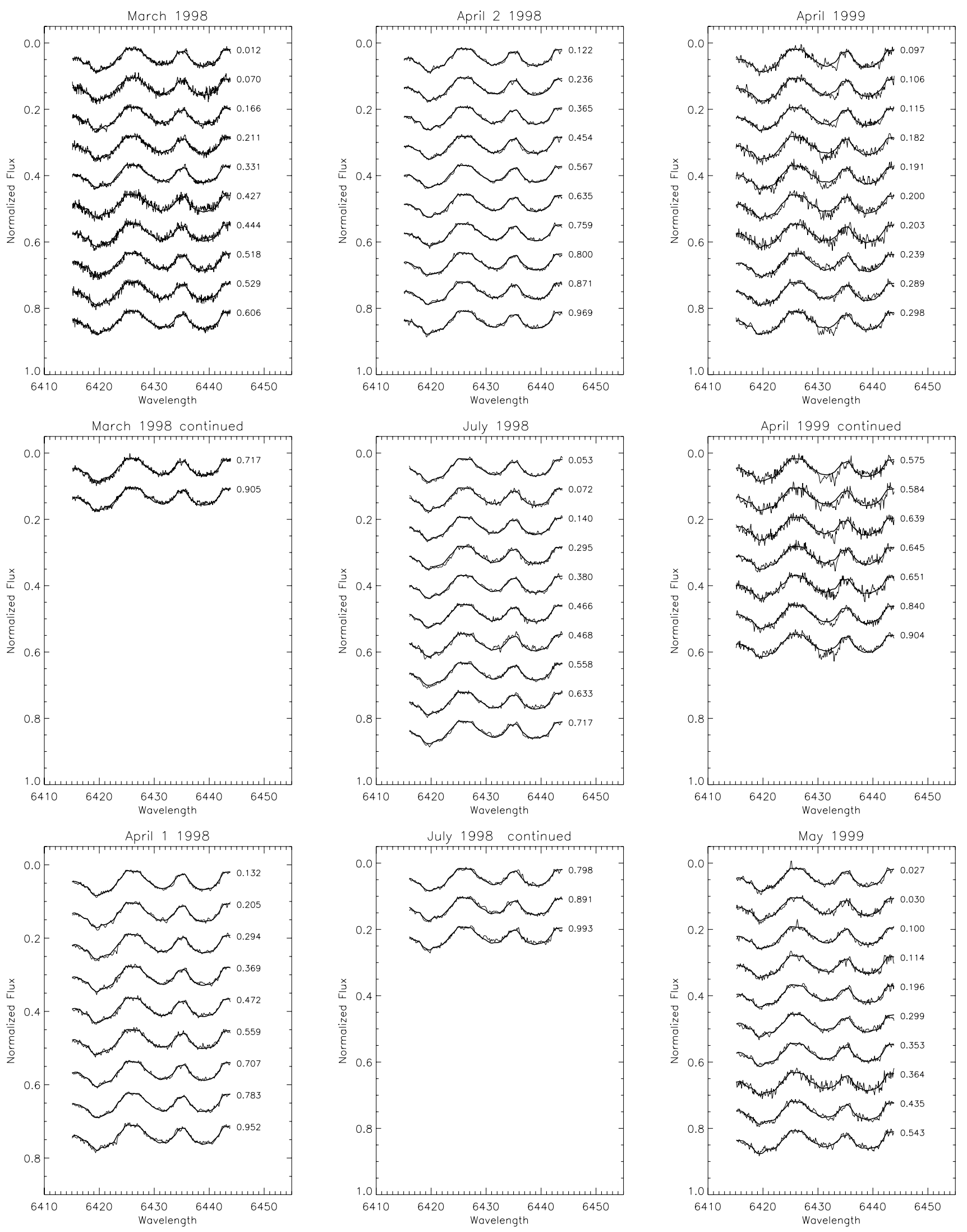

Fig. 2. continued. 
H. Korhonen et al.: Study of FK Comae Berenices. V., Online Material p 4
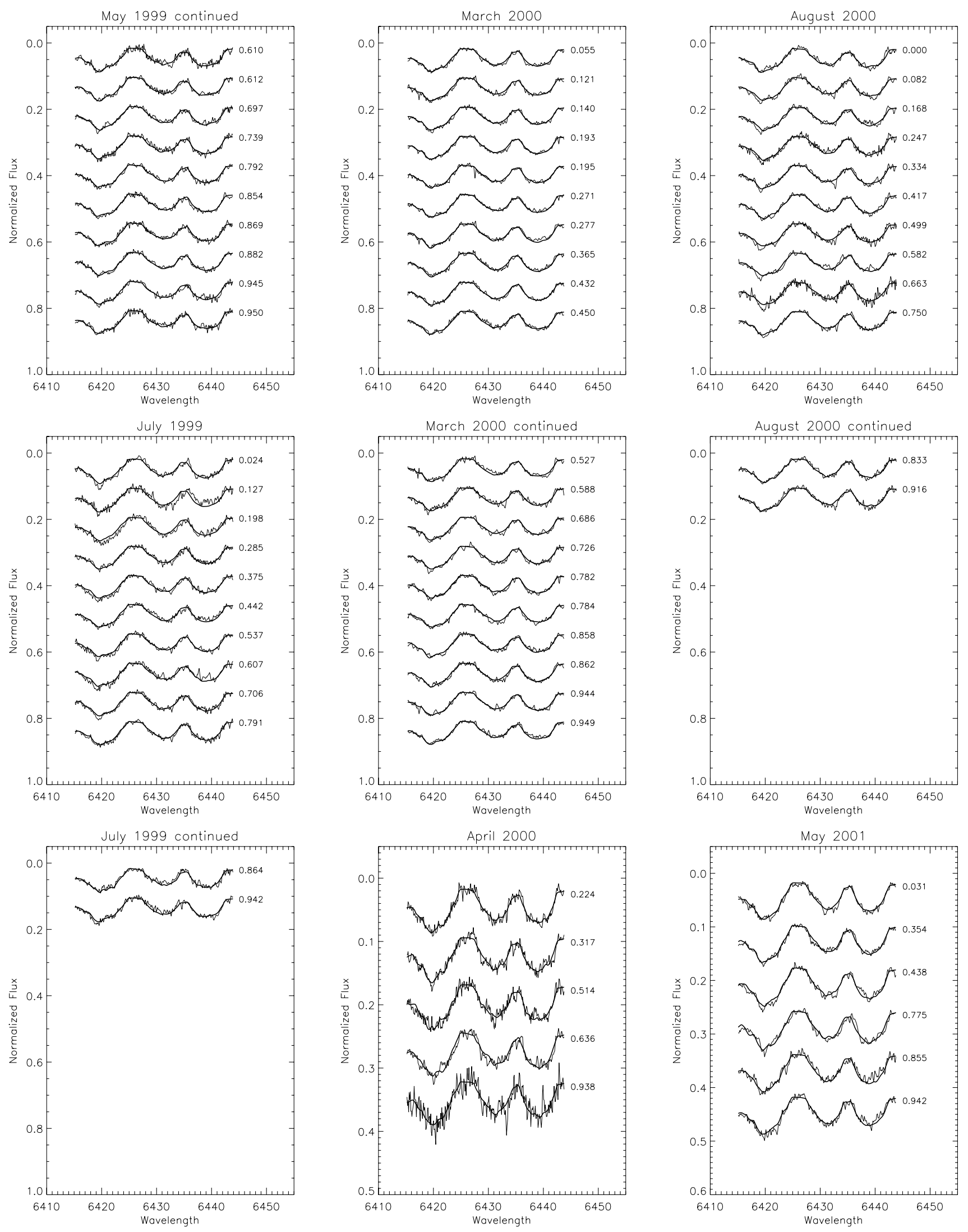

Fig. 2. continued. 
H. Korhonen et al.: Study of FK Comae Berenices. V., Online Material p 5
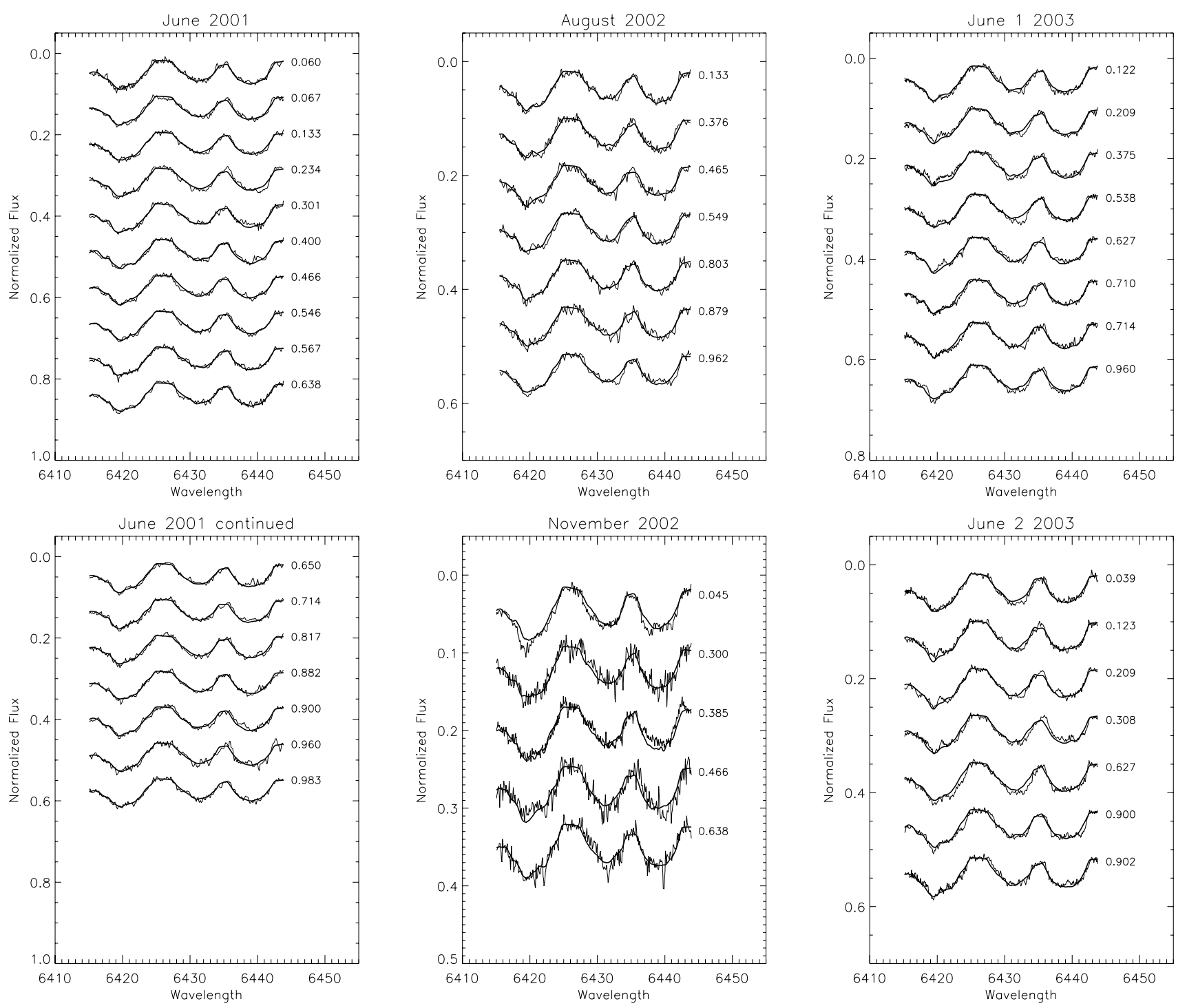

Fig. 2. continued. 
H. Korhonen et al.: Study of FK Comae Berenices. V., Online Material p 6
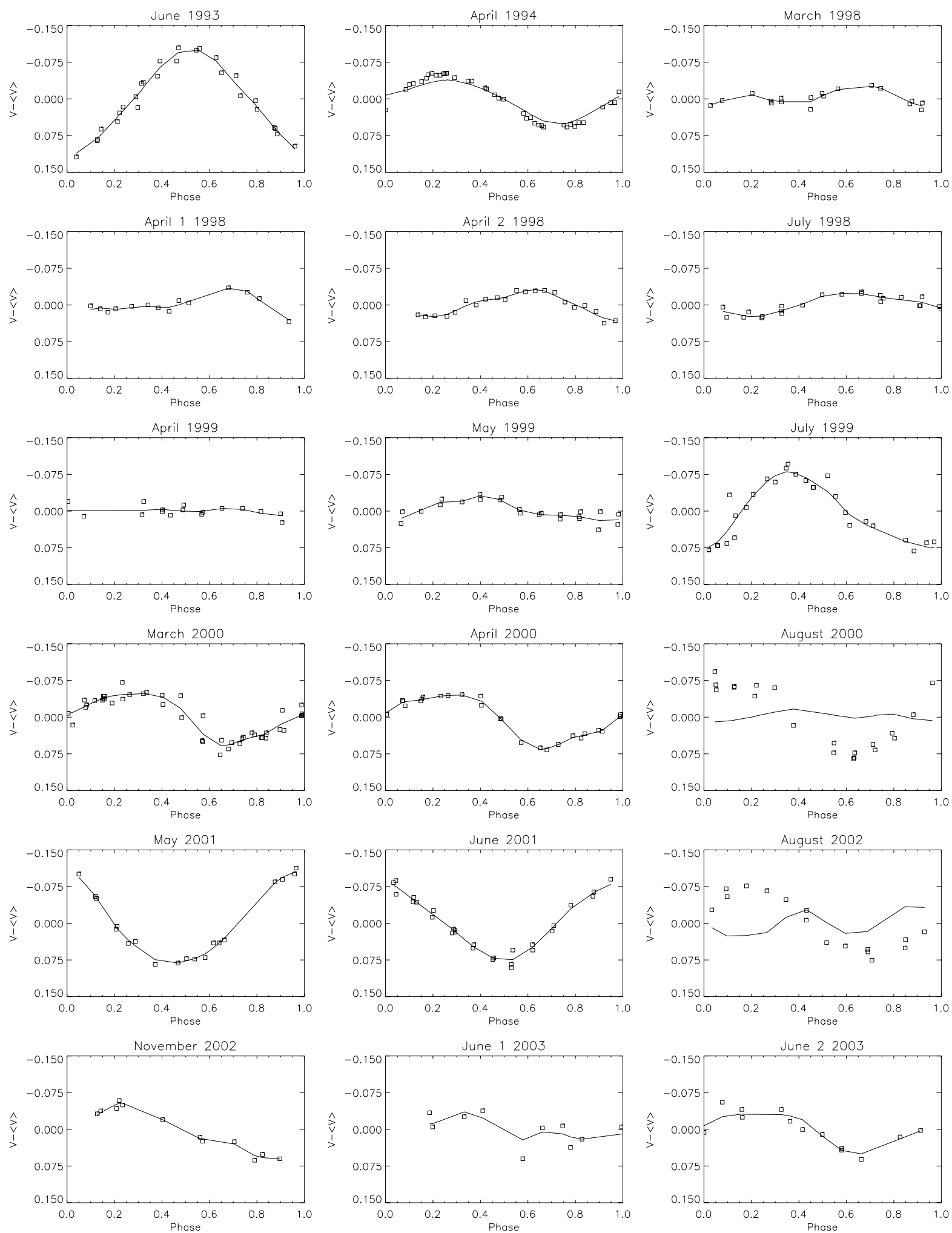

Fig. 3. The light-curves calculated from the temperature maps (solid line) together with simultaneous or near-simultaneous photometric observations (squares). Note that for all the maps, except for August 2000 and August 2002 for which no simultaneous photometry was available, the photometry has been used as a constraint in the inversion. 